\title{
Micro Integral Membrane Protein (MIMP), a Newly Discovered Anti-Inflammatory Protein of Lactobacillus Plantarum, Enhances the Gut Barrier and Modulates Microbiota and Inflammatory Cytokines
}

\begin{abstract}
Mingming Yina,g Xuebing Yan ${ }^{a}$ Wenhao Weng ${ }^{\mathrm{b}, \mathrm{c}}$ Yongzhi Yanga,f Renyuan Gao ${ }^{\mathrm{a}}$ Minfeng Liu ${ }^{\mathrm{h}}$ Cheng Pan ${ }^{\mathrm{a}}$ Qi Zhu ${ }^{\mathrm{a}}$ Hao Lia Qing Weid ${ }^{\mathrm{a}}$ Tongyi Shen ${ }^{\mathrm{a}}$ Yanlei Ma ${ }^{a, e, f}$ Huanlong Qin ${ }^{a}$

aDepartment of GI Surgery, Shanghai Tenth People's Hospital Affiliated to Tongji University, Shanghai, bDepartment of Clinical Laboratory, Yangpu Hospital, Tongji University School of Medicine, Shanghai, 'Center for Translational Medicine, Yangpu Hospital, Tongji University School of Medicine, Shanghai, 'Department of Pathology, Shanghai Tenth People's Hospital Affiliated to Tongji University, Shanghai, eDepartment of Colorectal Surgery, Fudan University Shanghai Cancer Center, Shanghai, 'Department of Oncology, Shanghai Medical College, Fudan University, Shanghai, 9Department of General Surgery, Anhui NO.2 Province People's Hopital, Hefei, ' Department of Hepatobiliary Surgery, Affiliated Wuxi No.3 Hospital, Nantong University, Wuxi, China
\end{abstract}

\section{Key Words}

Inflammatory bowel disease $\cdot$ MIMP • Gut barrier • Microbiota $\bullet$ Inflammatory cytokines

\begin{abstract}
Background/Aims: Recent studies have demonstrated that the manipulation of the gut microbiome represents a promising treatment for inflammatory bowel disease (IBD). We previously identified micro integral membrane protein (MIMP) as the smallest domain of surface layer protein from Lactobacillus Plantarum. However, the therapeutic relevance of MIMP in IBD remains unknown. Methods: We initially employed a dextran sodium sulphate (DSS)-induced colitis model and evaluated the effect of MIMP on the inflammation response, intestinal barrier and gut microbiota using histological examination, Fluorescein isothiocyanateDextran detection and pyrosequencing analysis respectively. We then established peripheral blood mononuclear cells (PBMCs) and an epithelial CaCO-2 co-culture model to investigate the regulatory role of MIMP in inflammatory cytokines. The level changes of inflammatory cytokines were detected using Enzyme-linked immunosorbent and real-time polymerase chain reaction assay. The involved regulatory mechanisms were investigated mainly using dual luciferase reporter and chromatin immunoprecipitation assay. Results: In the DSSinduced colitis model, we observed that MIMP intervention effectively improved the body

M. Yin, X. Yan and W. Weng contributed equally to this work.

Huanlong Qin, Yanlei Ma and Tongyi Shen

Department of GI Surgery, Shanghai Tenth People's Hospital Affiliated to Tongji University, Department of Colorectal Surgery and Department of Oncology, Fudan University, Shanghai (China); E-Mail hl-qin@hotmail.com, yanleima@live.cn, stonesty@sina.com
\end{abstract}




\section{Cellular Physiology Cell Physiol Biochem 2018;45:474-490 \\ \begin{tabular}{ll|l} 
DOI: 10.1159/000487027 & O 2018 The Author(s). Published by S. Karger AG, Basel \\
and Biochemistryer.com/cpb
\end{tabular} \\ Yin et al.: MIMP Regulates Gut Barrier, Mcrobiota and Inflammatory Cytokines}

weight loss, increased the colon length and decreased disease activity index. Consistently, the inflammation scores in the MIMP treatment group were significantly lower than those in the DSS treatment group. Furthermore, MIMP intervention was found to successfully neutralize DSS treatment by decreasing the expression of pro-inflammatory cytokines (IFN- $\gamma$, IL-17 and IL-23) and increasing the expression of anti-inflammatory cytokines (IL-4 and IL-10). Notably, the permeability assay demonstrated that the MIMP treatment group was remarkably lower than that in the DSS treatment group. We also showed that MIMP improved gut microbiota dysbiosis caused by DSS-induced inflammation. Additionally, in PBMCs and the CaCO-2 coculture model, MIMP showed an obvious suppressive effect on lipopolysaccharide-induced inflammation in a time- and dose-dependent manner. Furthermore, we revealed that MIMP could modulate inflammatory cytokine expression through the toll-like receptor 4 pathway and histone acetylation. Conclusions: Our results suggested that MIMP showed a significant anti-inflammatory effect through regulating the gut barrier, microbiota and inflammatory cytokines. MIMP may have translational relevance as clinically relevant therapy for IBD patients.

\section{Introduction}

(C) 2018 The Author(s)

Published by S. Karger AG, Basel

Inflammatory bowel disease (IBD), including ulcerative colitis (UC) and Crohn' disease (CD), is characterized by chronic and relapsing inflammation of the gastrointestinal tract. The incidence has been increasing rapidly in Asian countries, particularly in China, during the past decades [1]. The pathogenesis of IBD is still largely unknown, although several studies have suggested that genetic and environmental factors may contribute to the development of IBD[2].

Currently, UC patients usually receive 5-aminosalicylic acid (5-ASA)-based therapy, while most CD patients eventually undergo abdominal surgery. Unfortunately, it was shown that these treatments have limited clinical efficacy and a high incidence of side effects [3]. Although recent studies have reported that biologic therapies, such as anti-TNF treatment, hold promise for refractory IBD patients, nearly $30 \%$ of patients will develop drug resistance during treatment [4]. Therefore, a novel therapeutic target and treatment strategy for IBD are urgently needed.

Accumulating evidence has demonstrated that microbial dysbiosis plays a crucial role in the pathogenesis of IBD through affecting the metabolism pathway, intestinal barrier and immunity system. Therefore, the therapeutic manipulation of dysbiotic microbiota has been widely used for the treatment of IBD and has been proven to be a promising therapy with significant clinical efficacy for such a disease [5]. Our previous studies have shown that Lactobacillus Plantarum (LP) functions as a probiotic by enhancing the intestinal epithelial barrier [6-8]. We also observed that LP interacts with dendritic cells and regulates several inflammatory cytokines [9]. Clinically, we confirmed that perioperative probiotic treatment can reduce the rate of postoperative septicaemia and is associated with reduced serum concentrations of the intestinal permeability regulator zonulin in colorectal cancer patients undergoing colectomy [10]. Accordingly, to further investigate the functional role of LP, we extracted the surface layer protein (SLP) from LP. Surprisingly, we found that SLP could significantly alleviate intestinal epithelial cells from enteropathogenic Escherichia coli (EPEC)-induced injury [11]. We further identified micro integral membrane protein (MIMP) as the smallest domain of SLPs and showed its role in the protection of intestinal epithelial cells from EPEC-induced injury [12-16].

Despite our accumulated knowledge of MIMP, the mechanisms underlying the beneficial role of MIMP in IBD remain largely unknown. Therefore, we aimed to systematically and comprehensively investigate the functional contributions of the Lactobacillus Plantarum component MIMP in in vivo and in vitro models of IBD, identify specific mechanisms responsible for its beneficial effect during colitis and decipher whether MIMP may have translational relevance as a clinically relevant therapeutic target for IBD patients. 


\section{Cellular Physiology Cell Physiol Biochem 2018;45:474-490 and Biochemistry Publisned onlIne: January 31, 2018 \begin{tabular}{l|l} 
DOI: 10.1159/000487027 & $\begin{array}{l}\text { (c) } 2018 \text { The Author(s). Published by S. Karger AG, Basel } \\
\text { www.karger.com/cpb }\end{array}$
\end{tabular}}

Yin et al.: MIMP Regulates Gut Barrier, Mcrobiota and Inflammatory Cytokines

\section{Materials and Methods}

\section{Cell culture and Reagents}

CaCO-2 cells were grown in DMEM with 10\% foetal bovine serum (FBS, Invitrogen, USA), $100 \mathrm{U} /$ $\mathrm{mL}$ penicillin and $100 \mu \mathrm{g} / \mathrm{mL}$ streptomycin at $37^{\circ} \mathrm{C}$ in a $95 \%$ humidified atmosphere containing $5 \% \mathrm{CO}_{2}$. Human peripheral blood mononuclear cells (PBMCs) were prepared as described previously [17]. Briefly, the samples of peripheral blood from healthy volunteers were collected in heparinized vacutainers and then were processed to isolate PBMCs by density gradient centrifugation using lymphoprep (Axis-Shield PoC AS, Norway). The acquired PBMCs were washed twice with PBS solution containing 5\% FBS before transferring to the co-culture system. The study was approved by the ethics committee of Shanghai Tenth People's Hospital Affiliated to Tongji University, and written informed consent was obtained from healthy volunteers to use their blood samples in scientific studies.

Recombinant MIMP was biosynthesized and identified as described in our previous study [12]. The DSS reagent was purchased from MP Biomedicals (USA). Fluorescein isothiocyanate-Dextran and lipopolysaccharide (LPS) were purchased from Sigma (USA). Enzyme-linked immunosorbent assays (ELISA) kits for IL-4, IL-10, IFN- $\gamma$, IL-17 and IL-23 quantification were purchased from Cell Sciences (USA). All the primary antibodies were purchased from Abcam (USA). Pierce Agarose ChIP Kits were purchased from Thermo (USA). TLR4 antagonist was purchased from InvivoGen (Toulouse, France).

\section{DSS-induced colitis Model}

The colitis model was established by DSS induction according to a previous description [18]. Briefly, we divided eighteen 10-week-old male C57BL6 mice into 3 groups: healthy control group $(n=6)$, DSStreated group ( $\mathrm{n}=6)$, and MIMP-treated group $(\mathrm{n}=6)$. DSS-treated mice were administered drinking water containing 2.5\% DSS for 7 consecutive days. MIMP-treated mice were first administered with MIMP 0.1 $\mu \mathrm{g} / 20 \mathrm{~g}$ ) for 7 days before DSS induction and then were continuously administered the drug for 7 days [19]. For colitis assessment, the disease activity index (DAI) was employed and calculated based on weight loss percentages, diarrhoea and haematochezia. After MIMP intervention for 14 days, the mice were sacrificed, and their faeces and colon samples were harvested and stored in liquid nitrogen. All procedures were manipulated according to protocols approved by the Animal Care and Use Committee of Shanghai Tenth People's Hospital affiliated to Tongji University.

\section{Haematoxylin-eosin staining and histopathology evaluation}

Paraffin-embedded colon tissues were cut into 5-mm sections. The sections were dewaxed in xylene for $10 \mathrm{~min}$ and dehydrated in gradient alcohol. The sections were then stained with haematoxylin for $8 \mathrm{~min}$ and eosin for $5 \mathrm{~min}$. After dehydration, the sections were sealed and examined under a light microscope (Nikon Eclipse 80i, Japan). The histopathology evaluation was performed by two researchers who were blinded to the details of each section. The evaluation criteria were performed as follows: score 0 , no inflammation; score 1, moderate inflammatory infiltration in the basal layer; score 2, moderate mucosal hyperplasia or severe inflammatory infiltration; score 3 , severe mucosal hyperplasia; score 4, crypt absence or ulceration.

\section{Intestinal permeability assay}

The intestinal permeability was measured using the FITC-Dextran method. In brief, after fasting for $6 \mathrm{~h}$, the mice were given FITC-Dextran $(0.6 \mathrm{mg} / \mathrm{g}$, Sigma, USA) by oral gavage for $1 \mathrm{~h}$, and their peripheral blood was collected. The blood samples were then centrifuged at $3000 \mathrm{r} / \mathrm{min}$ to isolate the plasma. The obtained plasma was mixed with PBS solution at an equal volume. Finally, the concentration of FITC in the samples was detected by a microplate reader (Leica, Germany).

\section{Pyrosequencing analysis and bioinformatics analysis}

The DNA of the faeces samples was extracted using the QIAquick gel extraction kit (Qiagen, Germany) and was quantified using a NanoDrop ND-1000 spectrophotometer (Thermo Scientific, USA). The V3-V4 region of the bacterial $16 \mathrm{~S}$ rRNA gene was amplified in triplicate using primers 27F (5'-AGAGTTTGATCCTGGCTCAG-3') and 533R (5'-TTACCGCGGCTGCTGGCAC-3'). Twenty-microliter reactions were sequenced using a 454 Life Sciences GS FLX System with standard chemistry by the University of South Carolina's Engencore Sequencing Facility. 


\section{Cellular Physiology Cell Physiol Biochem 2018;45:474-490 \begin{tabular}{ll|l} 
DOI: 10.1159/000487027 & O 2018 The Author(s). Published by S. Karger AG, Basel \\
www.karger.com/cpb
\end{tabular} \\ Yin et al.: MIMP Regulates Gut Barrier, Mcrobiota and Inflammatory Cytokines}

Raw pyrosequencing reads obtained from the sequencer were denoised using Titanium PyroNoise software. Low-quality reads were identified and removed, and high-quality reads for bioinformatics analysis were selected; all the effective reads were clustered into OTUs based on $97 \%$ sequence similarity according to Usearch (version $7.1 \mathrm{http}: / /$ qiime.org/). Chimera sequences from the PCR amplification were detected and excluded from the OTUs using uchime (version 4.2.40http://drive5.com/usearch/manual/uchime_ algo.html). Alpha diversity (Shannon, Simpson) and richness (ACE and Chao) were analysed using QIIME69. The heatmap, PCoA and PCA analysis were performed on the relative abundance of OTUs using R (version 2.15; The R Project for Statistical Computing, http://www.R-project.org). Phylogenetic beta diversity such as hierarchical clustering analysis were determined by the OTUs using QIIME69. Taxonomy-based analyses were performed using a Bayesian classifier with a homemade RDP database (http://rdp.cme.msu.edu/) with a $50 \%$ bootstrap score. The microbiome features of the healthy controls were compared with those of mice with IBD using the $\mathrm{P}$ value and false discovery rate ( $\mathrm{Q}$ value) for nonnormal distributions. Only taxa with an average abundance $>1 \%$, $P$ value $<0.05$, and $Q$ value $<0.05$ (i.e., low risk of false discovery) were considered significant. The linear discriminant analysis (LDA) effect size (LEfSe) method was used to identify the distinctive microfloras significantly influenced by sample grouping.

\section{Dose- and time-response assays}

The co-culture system based on a 12-well transwell (Corning, USA) was utilized for dose- and timeresponse assays. PBMCs $\left(2 \times 10^{5}\right.$ cells/well) were cultured in the apical compartment, while CaCO- 2 cells $\left(2 \times 10^{6}\right.$ cells $/$ well) were cultured in the basolateral compartment. All the assays were performed after treating PBMCs or CaCO-2 cells with LPS $(100 \mathrm{ng} / \mathrm{ml})$ for $2 \mathrm{~h}$. For the dose-response assay, the apical compartment was supplemented with medium alone (control group) or medium containing gradient concentrations of $\operatorname{MIMP}(0.001,0.01,0.1,1,10,100,1000,10000 \mathrm{ng} / \mathrm{ml})$ for $48 \mathrm{~h}$. For the time-response assay, the CaCO- 2 cells were supplemented with medium containing $1 \mathrm{ng} / \mathrm{ml}$ MIMP for 3, 6, 9, 12, 24 and $48 \mathrm{~h}$. For the subsequent detection of inflammatory cytokines, the supernatants of both the apical and basolateral compartments were collected and centrifuged to remove cells at $400 \mathrm{~g}$ for $10 \mathrm{~min}$.

\section{RNA extraction, semi-quantitative and quantitative real-time PCR}

Total RNA from colon tissues and cells was extracted using the Trizol reagent (Gibco, USA) and RNeasy Mini kit (Qiagen, Germany) according to the manufacturer's instructions. The quantity and quality of RNA were assessed by the ratio of the absorbance values at 260 and $280 \mathrm{~nm}$. For semi-quantitative RT-PCR, 5 $\mu \mathrm{g}$ of total RNA was reversely transcribed into cDNA using the SuperScriptTM first-strand synthesis system (Invitrogen, USA), and then $1 \mu \mathrm{L}$ of cDNA product was applied for PCR amplification. Finally, the PCR products were analysed on $1.5 \%$ agarose gels. For quantitative RT-PCR, after amplification, PCR was performed using the LightCycler@ 480 Real-Time PCR System (Roche, Switzerland) and the QuantiFast SYBR Green PCR Kit (Qiagen, Germany). $\beta$-actin was employed as an internal control, and the relative expression of genes was calculated using $2^{-\Delta \Delta \mathrm{T}}$ method. The primers for the genes are listed (for all online suppl. material, see www. karger.com/doi/10.1159/000487027) in Table S1.

\section{Western blotting}

Total protein of tissues and cells lysates was extracted using lysis buffer, subjected to sodium dodecyl sulphate-polyacrylamide gel electrophoresis (SDS-PAGE) and transferred onto PVDF membranes. The membranes were incubated with the following antibodies (1:1000) overnight: anti-JAM-1 (Abgent Cat\# ab52647, RRID:AB_881206), anti-Occludin (Abgent Cat\# LS-B2895-50,RRID:AB_1935951), anti-ZO-1 (Abgent Cat\# ab96594, RRID:AB_10678863), anti-ERK1/2 (Abgent Cat\# 4695P, RRID: AB_390779), anti-pERK1/2 (Abgent Cat\# 4370P,RRID:AB_2315112), anti-p38 (Abgent Cat\# 8690P, RRID:AB_10998134), antip-p38 (Abgent Cat\# 4511S, RRID: AB_2139682), anti-JNK (Abgent Cat\# 9252, RRID:AB_2250373), anti-pJNK (Abgent Cat\# 4668S, RRID:AB_823588), anti-IkB $\alpha$ (Abgent Cat\# 9242L,RRID: AB_823540), anti-IRAK1 (Abgent Cat\# 10478-2-AP, RRID:AB_2126032), anti-MyD88 (Abgent Cat\# ab133739, RRID:AB_2637005), anti-p65 (Abgent Cat\# 4764, RRID:AB_823578), anti-p50 (Abgent Cat\# ab32360, RRID:AB_776748), anti-H3 (Abgent Cat\# ab176842, RRID:AB_2493104), anti-Ac-P-H3 (Abgent Cat\# 07-081, RRID:AB_310366), anti-H4 (Abgent Cat\# ab10158, RRID:AB_296888), anti-Ac-H4 (Abgent Cat\# ab45166, RRID:AB_732937), antiGAPDH (Abgent Cat\# ab9483, RRID: AB_307273) and anti- $\beta$-actin (Abgent Cat\# 1854-1, RRID:AB_764434). After incubation with secondary antibody $(1: 5000)$ for $1 \mathrm{~h}$, the protein expression was visualized using the enhanced chemiluminescence (ECL) method. 


\section{Cellular Physiology Cell Physiol Biochem 2018:45:474-490 and Biochemistry Publisned onIme: January 31, 2018 \begin{tabular}{l|l} 
DOI: 10.1159/000487027 2018 The Author(s). Published by S. Karger AG, Basel \\
www.karger.com/cpb
\end{tabular}}

Yin et al.: MIMP Regulates Gut Barrier, Mcrobiota and Inflammatory Cytokines

Enzyme-linked immunosorbent assay

The levels of inflammatory cytokines in the cell-free supernatants of the co-culture system were detected using ELISA kits according to the manufacturer's instructions. The plates were read at $450 \mathrm{~nm}$ and the obtained data were processed to calculate the concentrations of inflammatory cytokines using standard curves.

\section{Dual luciferase reporter assay}

For the transcription activity assay, CaCO-2 cells were transfected with a luciferase reporter plasmid at $100 \mathrm{ng} /$ well, and the internal control plasmid (pRL-TK) at $9.6 \mathrm{ng} /$ well using the FuGENEHD Transfection Reagent (Promega, USA). After removing the medium, the cells were washed with PBS and supplemented with fresh complete medium. Next, the cells were treated with $100 \mathrm{ng} / \mathrm{ml}$ LPS and MIMP for gradient concentrations $(0.01 \mathrm{ng} / \mathrm{ml}, 0.1 \mathrm{ng} / \mathrm{ml}$ and $1 \mathrm{ng} / \mathrm{ml})$ or gradient incubation period $(3 \mathrm{~h}, 6 \mathrm{~h}, 9 \mathrm{~h}$ and $12 \mathrm{~h})$. Subsequently, the CaCO-2 cells were lysed and $20 \mu \mathrm{L}$ lysates were incubated with $50 \mu \mathrm{L}$ Luciferase Assay Buffer II (Promega, USA) in an opaque 96-well plate. The fluorescence values of firefly and Renilla luciferase were measured on a multimode plate reader (Tecan, Switzerland). The relative luciferase activity was calculated by normalizing the fluorescence value of firefly luciferase to the fluorescence value of Renilla luciferase.

\section{Histone modification assays}

The culture medium of CaCO-2 cells was replaced by PBS solution containing $1 \%$ formaldehyde. $10 \times$ Glycine solution was applied for blocking crosslinking and the cells were suspended in ice-cold PBS. The cells were then lysed in $0.25 \mu \mathrm{l}$ Micrococcal Nuclease for $15 \mathrm{~min}$, incubated with $10 \mu \mathrm{L}$ MNase Stop Solution for $15 \mathrm{~min}$, centrifuged at $9000 \mathrm{~g}$ for $3 \mathrm{~min}$ and suspended in $50 \mu \mathrm{l}$ Lysis Buffer. Finally, the lysate was centrifuged at $9000 \mathrm{~g}$ for $3 \mathrm{~min}$ again and the supernatant (chromosome) was collected. The supernatant was incubated with $4 \mu \mathrm{l} \mathrm{IgG}, 5 \mu \mathrm{l}$ anti-AC-H4, $4 \mu \mathrm{l}$ anti-p-Ac-H3 and $2 \mu \mathrm{l}$ anti-RNA polymerase II overnight at overnight at $4^{\circ} \mathrm{C}$. The immune complexes were collected using proteinA Agarose/SalmonSperm DNA (Thermo) and the DNA was extracted using a PCR purification kit (Qiagen, Germany). The promoter DNA of IL-17 and IFN- $\gamma$ was amplified by PCR using Hot Star Taq DNA polymerase (Qiagen). The PCR reaction was conducted according to the following conditions: $95^{\circ} \mathrm{C}$ for $15 \mathrm{~min}, 34$ cycles of $94^{\circ} \mathrm{C}$ for $30 \mathrm{~s}, 60^{\circ} \mathrm{C}$ for 30 $\mathrm{s}, 72^{\circ} \mathrm{C}$ for $30 \mathrm{~s}$ and $72^{\circ} \mathrm{C}$ for $10 \mathrm{~min}$. The PCR products were separated by agarose gel electrophoresis and detected by ethidium bromide staining. Equal amounts of input DNA were employed as internal controls. The promoter-specific primers for IL-17 and IFN- $\gamma$ were listed, see online suppl. material, in Table S1. The levels of acetylated histones and methylated DNA were quantitatively assessed respectively using an EpiQuik Global Histone H3/H4 Acetylation Assay Kit and a Methylamp Global DNA Methylation Quantification Ultra Assay Kit (Epigentek Group, Farmingdale, NY, USA)

\section{Statistical analysis}

The data were presented as the mean \pm standard deviation (SD). All the statistical analysis were performed using 19.0 SPSS software (SPSS, USA). The qualitative variables were compared by chi-square test or Fisher's exact test, while the quantitative variables were compared by Student's t-test. A p value $<0.05$ (two-sided) indicated statistically significant.

\section{Results}

\section{MIMP alleviates inflammation response in DSS-induced colitis model}

The dextran sulphate sodium (DSS)-induced colitis model is widely used in IBD research [20]. This model was therefore employed to investigate the protective role of MIMP in IBD. We administered 2.5\% DSS or phosphate-buffered saline (PBS as a negative control, NC) into 8- to 10-week-old male C57BL6 mice for 7 consecutive days. For the MIMP treatment group, the administration of $0.1 \mu \mathrm{g} / 20 \mathrm{~g}$ MIMP was started 1 week before colitis induction and was continued throughout colitis development (Fig. 1A). Unfortunately, we found that 2 mice in the DSS-treated group and 1 mouse in the MIMP-treated group had died during DSS induction. We then used the remaining mice for subsequent experiments. In the DSSinduced colitis model, we observed decreased body weight and colon length compared with those in the control group ( $\mathrm{p}<0.001)$, suggesting that we successfully established the DSS- 


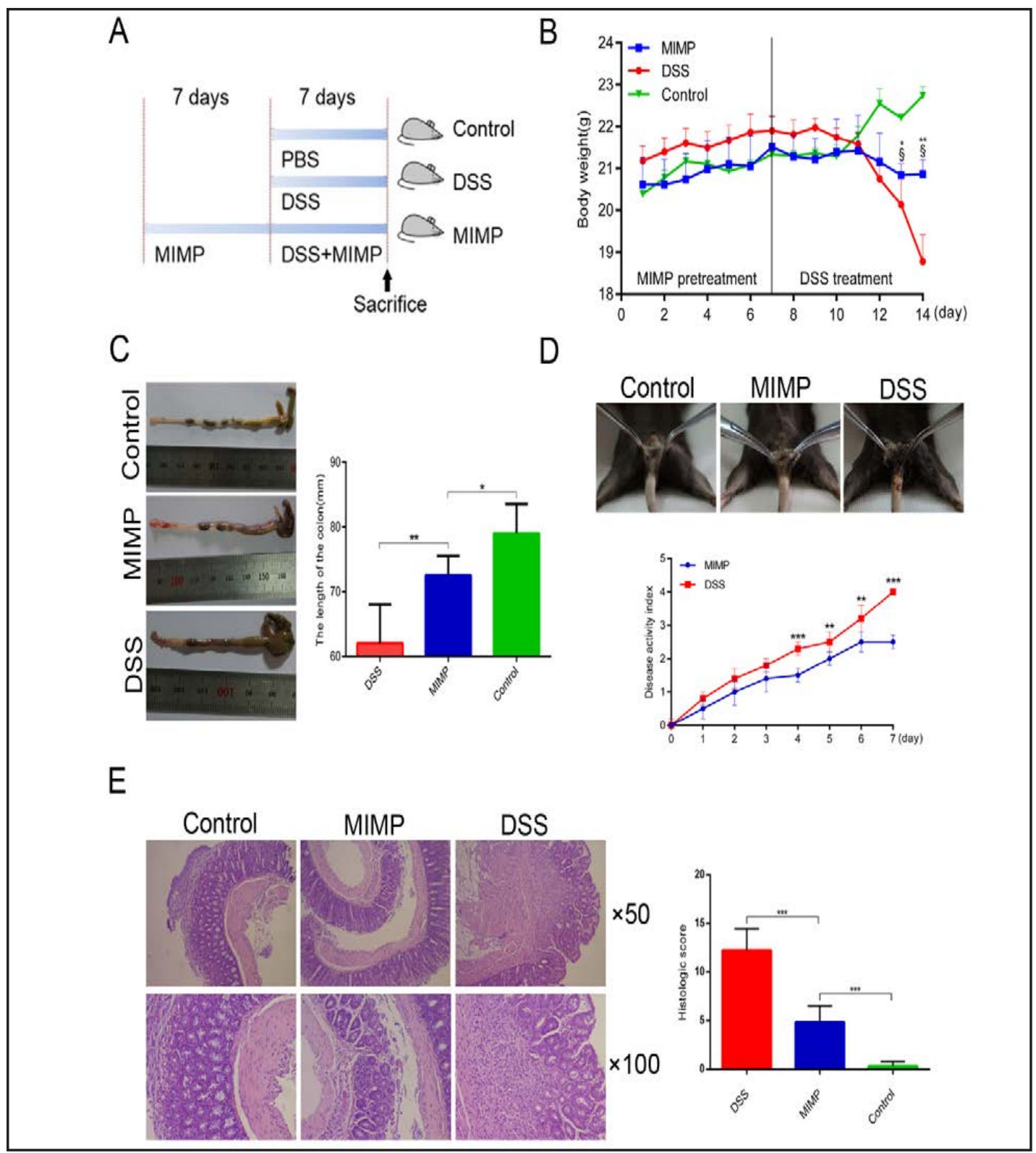

Fig. 1. MIMP alleviates the inflammation response in a DSS-induced colitis model(A) Diagram of MIMP intervention in the dextran sulphate sodium (DSS)-induced colitis model. (B) MIMP intervention improves the body weight loss caused by DSS treatment. (C) MIMP intervention increases the length of the colon in the DSS-induced colitis model. (D) MIMP intervention inhibits the colitis progression as evaluated by the disease activity index. (E) MIMP intervention decreases the inflammatory histological scores of the colon in a DSS-induced colitis model. The data are expressed as the mean $\pm \mathrm{SD}\left({ }^{*} \mathrm{P}<0.05 * * \mathrm{P}<0.01{ }^{* * *} \mathrm{P}<0.001\right.$, versus the control group; $\S \mathrm{P}<0.05$, versus the DSS group).

induced colitis model (Fig. 1B and C). Importantly, we observed that MIMP intervention could improve the body weight loss $(\mathrm{p}<0.05)$ and increase the colon length $(\mathrm{p}<0.01)$ caused by DSS treatment, indicating that MIMP can effectively alleviate intestinal inflammation (Fig. 1B and C). Furthermore, we calculated the disease activity index (DAI), based on weight loss, stool consistency, and bleeding, to evaluate the suppressive effect of MIMP on the progression of colitis. As shown in Fig. 1D, MIMP treatment began to exert its suppressive effect on the fourth day after DSS treatment $(\mathrm{p}<0.001)$. Consistent with DAI, our histopathology evaluation showed that the inflammation scores in the MIMP treatment group were significantly lower 
than those in the DSS treatment group ( $p<0.001$, Fig. 1E). Collectively, our data, for the first time, showed that MIMP can effectively alleviate the inflammation response in a DSS-induced colitis model.

\section{MIMP affects the expression of inflammatory cytokines and enhances the intestinal barrier} in the DSS-induced colitis model

Because our data showed that MIMP reduced the progression of colitis, we assumed that MIMP may have an impact on the expression of inflammatory cytokines during DSS induction. We measured the expression level of several anti-inflammatory cytokines (IL-4 and IL-10) as well as pro-inflammatory cytokines (IFN- $\gamma$, IL-17 and IL-23) in colon tissues from the control, DSS treatment and MIMP treatment groups. As shown in Fig. 2A, the expression levels of IFN- $\gamma$, IL-17 and IL-23 in DSS treatment group were significantly higher than those in the control group $(\mathrm{p}<0.01)$. Consistent with our assumption, MIMP intervention can dramatically decrease the expression of IFN- $\gamma$, IL-17 and IL-23 caused by DSS treatment $(\mathrm{p}<0.01)$. Similarly, DSS treatment results in the down-regulation of IL-4 and IL-10 compared with that in the control group $(\mathrm{p}<0.01)$, while MIMP can alleviate the suppression of IL- 4 and IL-10 by DSS treatment $(\mathrm{p}<0.01)$. Together, we found that MIMP regulates pro- and antiinflammatory cytokines to relieve DSS-induced inflammation.

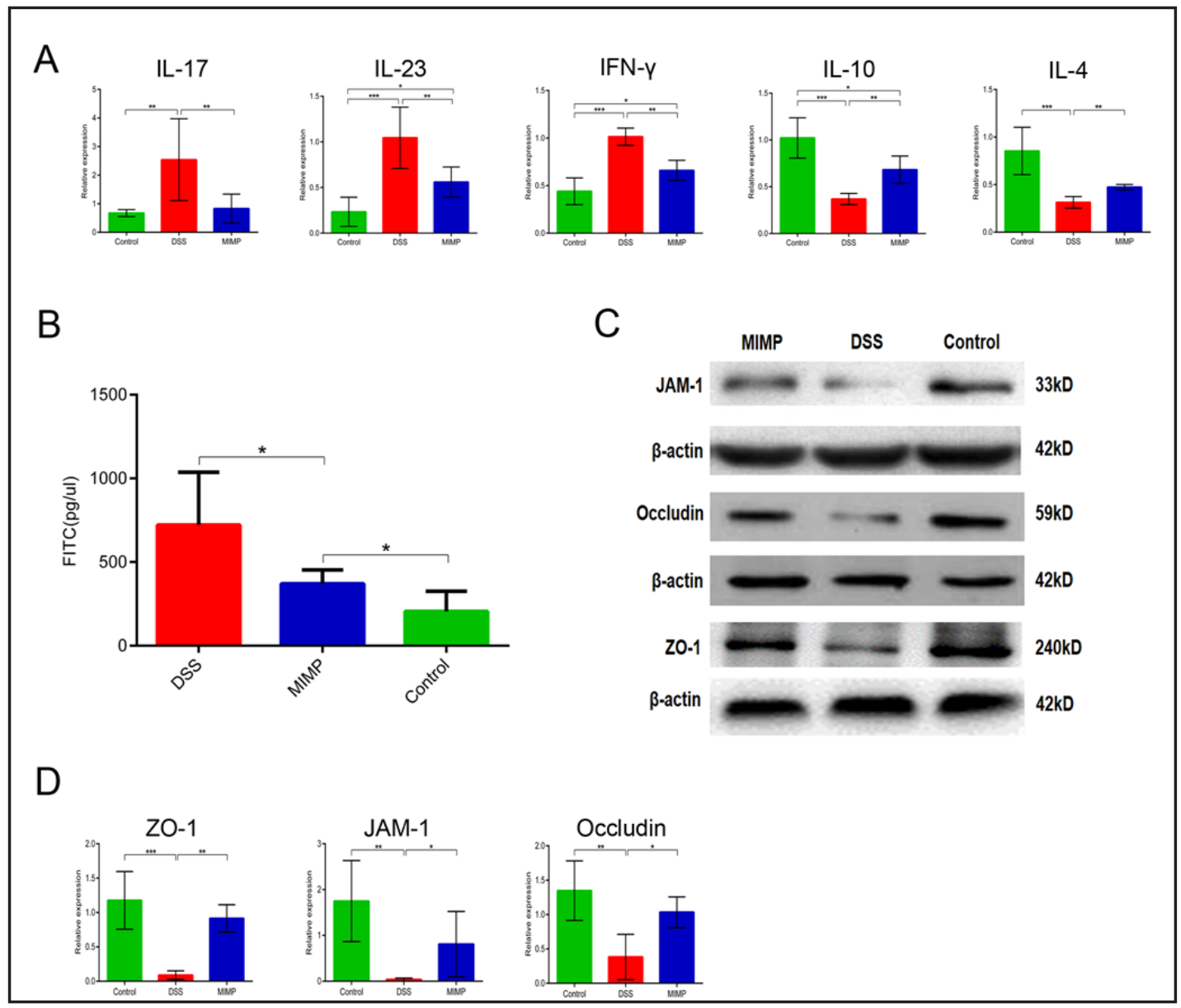

Fig. 2. MIMP alleviates the expression of inflammatory cytokines and enhances the intestinal barrier in the DSS-induced colitis model(A) MIMP intervention decreases the relative mRNA level of IL-17, IL-23, and IFN- $\gamma$ and increases that of IL-10 and IL-4 in the colon tissues of the DSS-induced colitis model. (B) MIMP intervention decreases the plasma concentration of FITC in the DSS-induced colitis model. (C-D) MIMP intervention up-regulates the expression of JAM-1, Occludin and ZO-1 in the colon tissues of the DSS-induced colitis model, as detected by western blotting (C) and RT-PCR (D). The data are expressed as the means \pm SD $\left({ }^{*} \mathrm{P}<0.05^{* *} \mathrm{P}<0.01^{* * *} \mathrm{P}<0.001\right)$. 


\section{Cellular Physiology Cell Physiol Biochem 2018:45:474-490

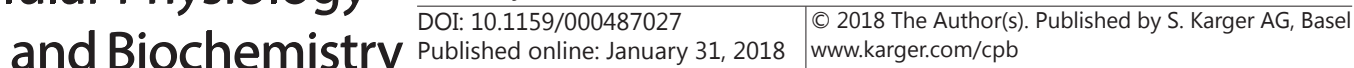

Our previous study demonstrated the protective role of MIMP in the intestinal barrier [16]. Hence, we assumed that MIMP may contribute to the resistance of DSS-induced inflammation through enhancing the gut barrier. We evaluated the intestinal permeability as measured by FITC-Dextran and found that the concentration of serum FITC in the MIMP treatment group was remarkably lower than that in the DSS treatment group $(370.91 \pm 82.78$ $\mathrm{pg} / \mu \mathrm{L} v s .722 .17 \pm 314.98 \mathrm{pg} / \mu \mathrm{L}, \mathrm{p}<0.05$, Fig. $2 \mathrm{~B}$ ), suggesting that MIMP, to some degree, inhibited the intestinal mucosal leakage by DSS-treatment. To further confirm our hypothesis, we measured the expression level of several tight junction proteins in colon tissues. Consistently, we observed that JAM-1, Occludin and ZO-1 were significantly upregulated by MIMP treatment compared with that by DSS treatment ( $\mathrm{p}<0.05$, Fig. $2 \mathrm{C}$ and D). Taken together, our results showed that MIMP improved intestinal barrier function during DSS-induced inflammation.

\section{DSS-induced gut microbiota dysbiosis is improved by MIMP}

There is growing evidence that gut microbiota dysbiosis is associated with IBD[21]. We hypothesized that MIMP may normalize dysbiosis that caused by DSS treatment and therefore reduce intestinal inflammation. We collected 15 stool samples, including 6 from the control group, 4 from the DSS treatment group and 5 from the MIMP treatment group. We subsequently performed pyrosequencing analysis of bacterial 16S rRNA (V3-V5 region) on these samples. In total, 378, 665 valid reads and 5, 087 Operational Taxonomic Units (OTUs) were acquired from these samples. The comparative analysis of the bacterial community abundance at the genus level in the 3 groups is shown, see online suppl. material, in Fig. S1. Although Chao and Ace did not show significant differences among the 3 groups ( $p>0.05)$, the Shannon and Simpson indexes clearly demonstrated the increasing community diversity from the DSS treatment group, MIMP treatment group to the control group (Fig. 3A).

Hierarchical cluster analysis was performed to show the structures of the bacterial communities in each group. As shown, see online suppl. material, in Fig. S2, a similar bacterial distribution was observed in the control and MIMP groups. Particularly, Bacteroidales_S247 was enriched in both the control and MIMP groups (56.84 and 37.65\%, respectively), but remarkably decreased in the DSS treatment group (17.24\%). Consistently, our PCoA analysis revealed that the microbiota compositions of the 3 groups were completely distinguished

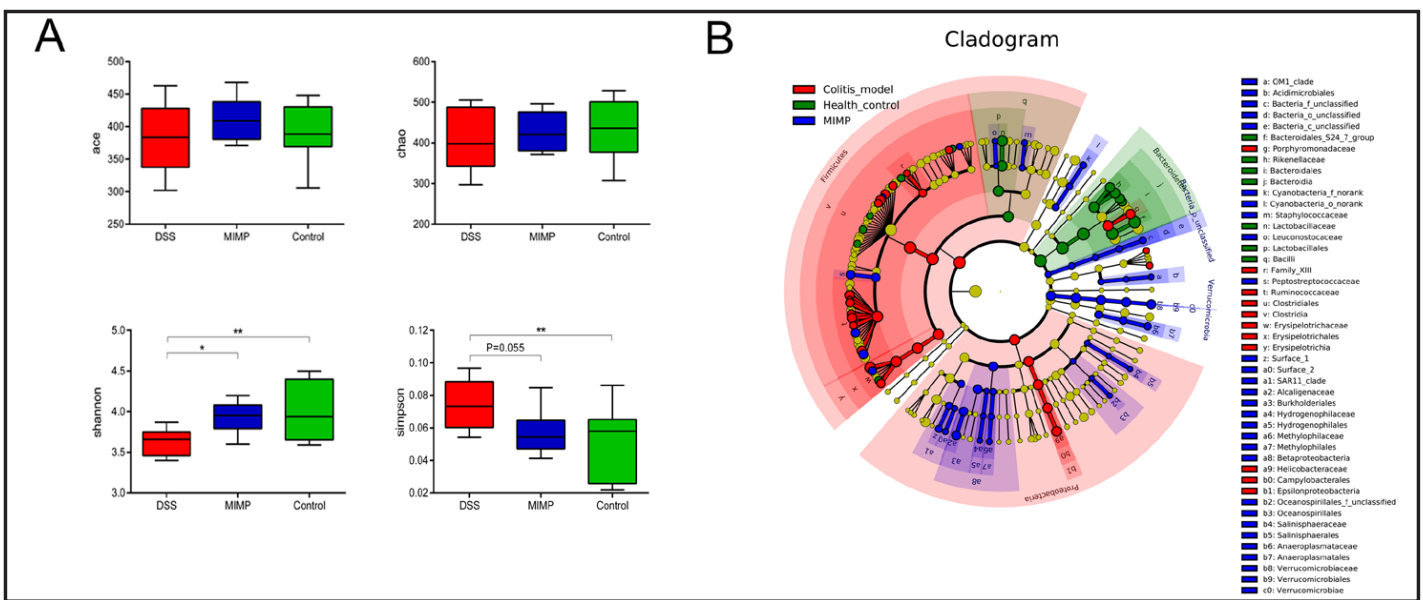

Fig. 3. DSS-induced gut microbiota dysbiosis is improved by MIMP(A) Richness and diversity analysis of gut microbiota. Upper panel: no statistical significance was found among the three groups in the richness analysis, as assessed by the ace index (left) and chao index (right). Bottom panel: MIMP intervention increases the diversity of gut microbiota in the DSS-induced colitis model, as assessed by the Shannon index (left, ${ }^{*} \mathrm{P}<0.05$ ) but not by the Simpson index (right, $\mathrm{P}=0.055$ ). (B) The Cladogram for differentially abundant gut microbiota in three group were constructed based on Lefse analysis. Firmicute and clostridia were enriched in the DSS-treatment group, while Leuconostocaceae and Leuconostoc were abundant in the MIMP group and Bacteroidales_S24-7 and Bacteroidales in the healthy control group. 
A
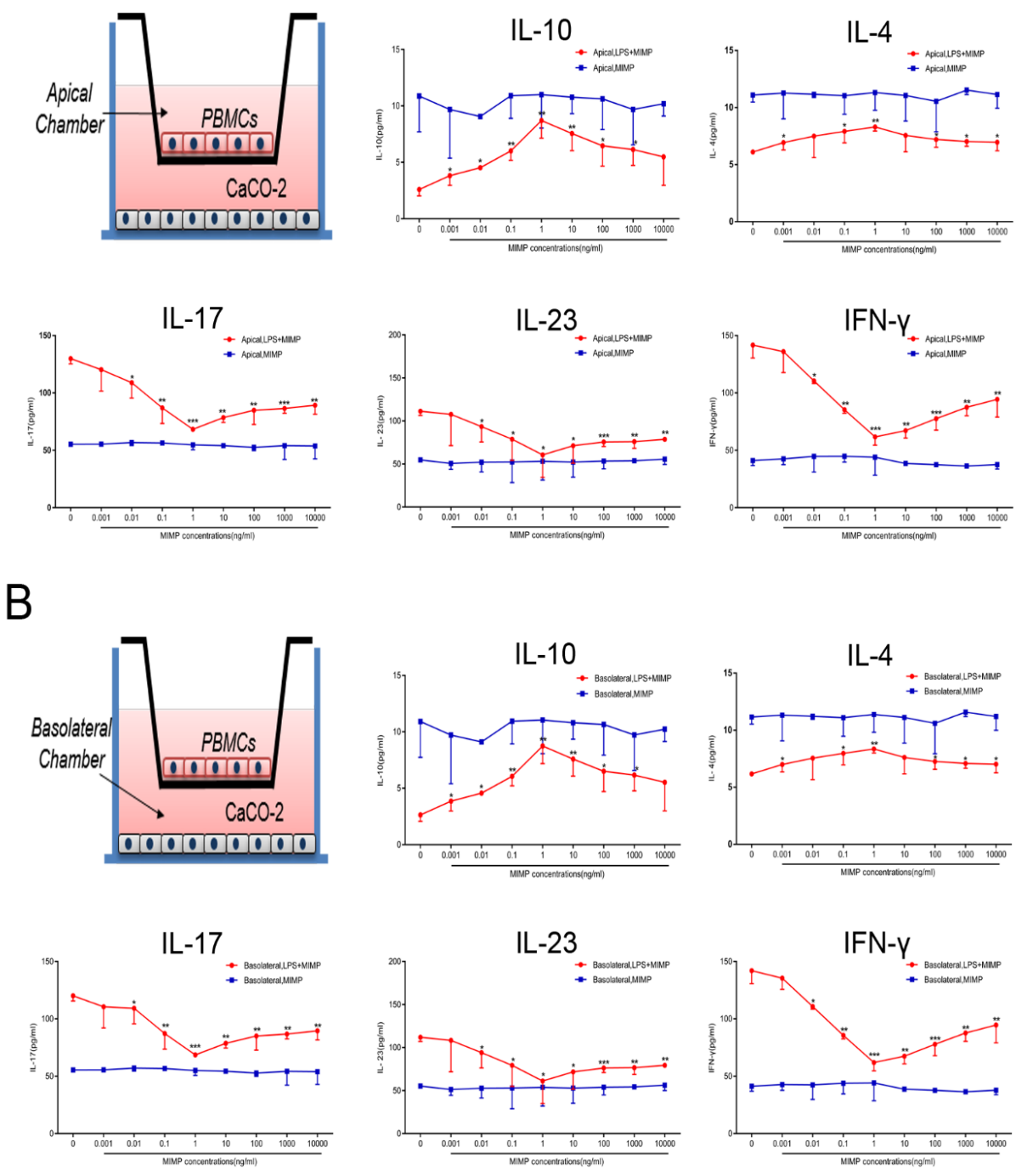

Fig. 4. MIMP exerts anti-inflammation activity through regulating the secretion of inflammatory cytokines by both PBMCs and epithelial CaCO-2 cells(A) For the PBMCs cultured in the apical compartment of the Transwell insert, MIMP promotes the secretion of IL-10, IL-4, and inhibits that of IL-17, IL-23 and IFN- $\gamma$ after LPS induction at a dose-dependent manner, with an optimal concentration of $1 \mathrm{ng} / \mathrm{ml}$. (B) For epithelial Caco- 2 cells cultured in the basolateral compartment of the Transwell insert, MIMP exerts a similar regulatory effect on the secretion of these inflammatory cytokines after LPS induction at the same dosedependent manner, with an optimal concentration of $1 \mathrm{ng} / \mathrm{ml}$. The data are expressed as the means \pm SD $\left({ }^{*} \mathrm{P}<0.05^{* *} \mathrm{P}<0.01 * * * \mathrm{P}<0.001\right)$.

by PC1 (66.66\%) and PC2 (13.75\%). Notably, the MIMP groups showed more similar microbiota composition in the control group than in DSS treatment group, as distinguished by PC1 (66.66\%) and PC3 (5.62\%, see online suppl. material, Fig. S3). Furthermore, the cladogram (Fig. 3B) and histogram of LDA scores (see online suppl. material, Fig. S4) revealed that Firmicute and clostridia were enriched in the DSS-treatment group, while Leuconostocaceae and Leuconostoc were abundant in the MIMP group and Bacteroidales S24-7 and Bacteroidales in the control group. Collectively, our results clearly showed that MIMP can improve gut microbiota dysbiosis caused by DSS-induced inflammation. 


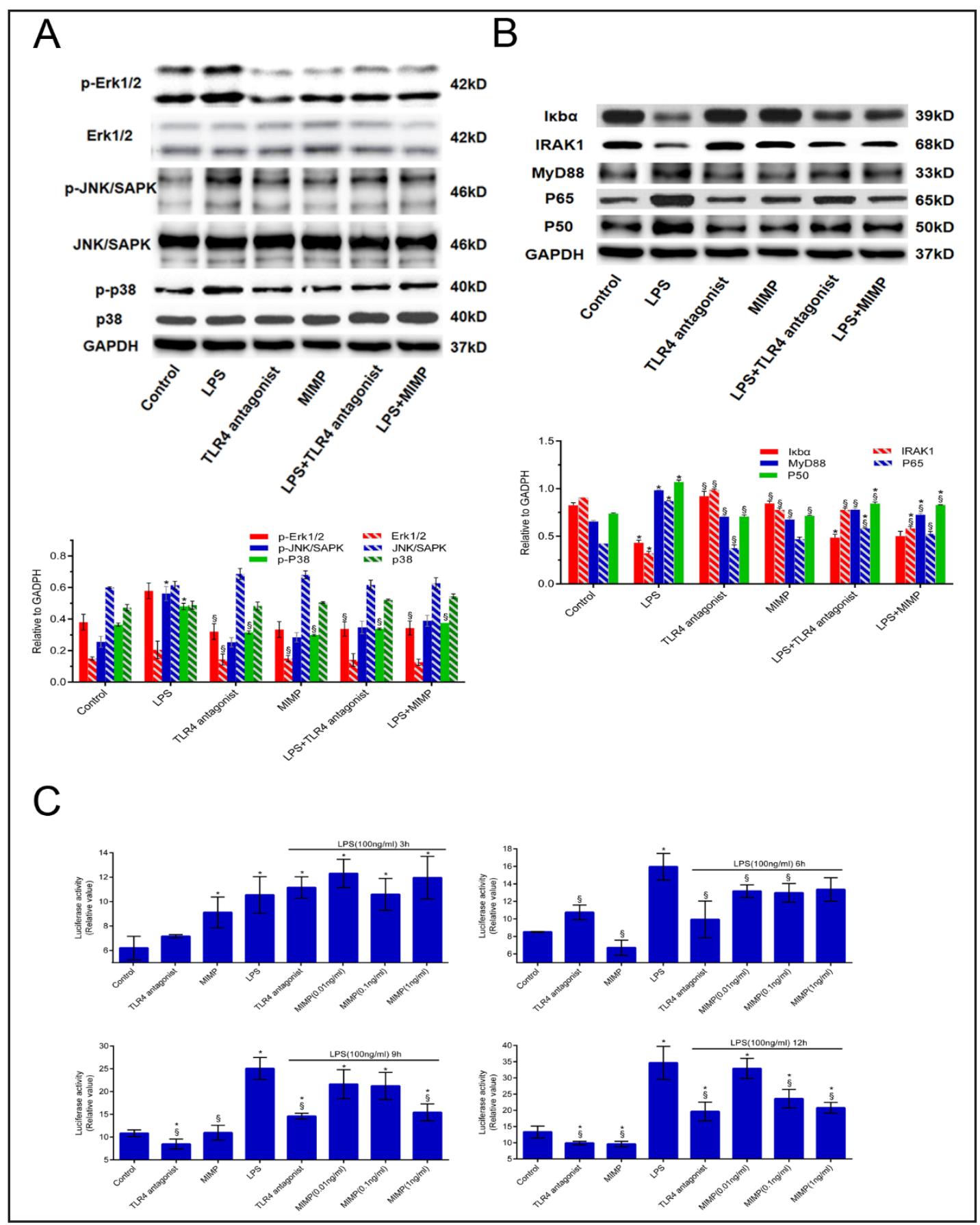

Fig. 5. MIMP regulates secretory inflammatory cytokines through the TLR4-related pathway.(A) MIMP and the TLR4 antagonist suppresses MAPK/JNK pathways activated by LPS in CaCO-2 cells. (B) MIMP and the TLR4 antagonist suppresses NF- $\kappa B$ pathways activated by LPS in CaCO-2 cells. (C) Compared with the TLR4 antagonist, MIMP begins to exert a suppressive effect on LPS-induced NF- $\mathrm{B}$ activity at $6 \mathrm{~h}, 9 \mathrm{~h}$ and $12 \mathrm{~h}$ after treatment, and functions in a dose-dependent manner at $9 \mathrm{~h}$ and $12 \mathrm{~h}$, with an optimal concentration of $1 \mathrm{ng} / \mathrm{ml}$. The data are expressed as the mean $\pm \mathrm{SD}$. * versus control; $\S$ versus LPS-stimulated cells $\mathrm{P}<0.05$.

MIMP exerts anti-inflammation activity through regulating the secretion of inflammatory cytokines by both PBMCs and epithelial CaCO-2 cells

It is well recognized that immune cells, as well as intestinal epithelial cells, are the main source of inflammatory cytokines [22]. Therefore, we established PBMCs and the CaCO-2 
co-culture model to decipher the mechanisms that MIMP contributes to anti-inflammation. In this co-culture model, PBMCs were grown on the filter membranes, and CaCO-2 cells were placed in the lower compartment of the culture plates (Fig. 4). Considering LPS as a strong in vitro inflammation inducer by far, we first treated PBMCs or CaCO-2 cells with LPS or MIMP (baseline) for $2 \mathrm{~h}$, and subsequently seeded treated cells in the upper or lower compartment of the culture plates supplemented with the medium alone (control) or medium with increasing concentrations of MIMP for $48 \mathrm{~h}$. In the control group (absence of MIMP), we observed that the levels of pro-inflammatory cytokines (IFN- $\gamma$, IL-17 and IL23 ) were significantly increased by LPS, while the levels of anti-inflammatory cytokines (IL4 and IL-10) were decreased, in both the apical and basolateral compartment, suggesting that LPS can effectively induce or suppress the secretion of inflammation cytokines in both PBMCs or CaCO-2 cells ( $p<0.01$, Fig. 4). Surprisingly, we found that MIMP showed an obvious inhibitory effect on LPS-induced inflammation with MIMP down-regulating the levels of pro-inflammatory cytokines (IFN- $\gamma$, IL-17 and IL-23) and up-regulating the levels of antiinflammatory cytokines (IL-4 and IL-10), with the increasing concentrations of MIMP. In particular, we found that $1 \mathrm{ng} / \mathrm{ml}$ of MIMP showed the maximal inhibitory effect, and we used this concentration in subsequent experiments. Furthermore, we measured the expression level of inflammation cytokines in PBMCs and CaCO-2 cells with different treatments and confirmed that the expression levels in PBMCs and CaCO-2 cells agreed with the level of secretory inflammation cytokines in the apical and basolateral compartment (see online suppl. material, Fig. S5), suggesting MIMP affects secretion of inflammation cytokines by regulating their expression in PBMCs and CaCO-2 cells.

We further questioned whether this inhibitory effect of MIMP on LPS treatment is time-dependent. Thus, we used $1 \mathrm{ng} / \mathrm{ml}$ of MIMP to perform the same experiments with different MIMP incubation times. As shown, see online suppl. material, in Fig, S6, MIMP produces a maximal inhibitory effect at least at $12 \mathrm{~h}$ after MIMP treatment. We also confirmed that the expression levels in PBMCs and CaCO-2 cells are consistent with those of secretory inflammation cytokines in apical and basolateral compartments (see online suppl. material, Fig. S7). Taken together, our results suggest that MIMP regulates the secretion of inflammatory cytokines by PBMCs and CaCO-2 cells during LPS-induced inflammation in a dose- and time-dependent manner. Such an effect of MIMP can effectively prevent the progression of inflammation by LPS.

\section{MIMP regulates secretory inflammatory cytokines through the TLR4 pathway and Histone acetylation}

We next interrogated the mechanistic role of MIMP in the regulation of secretory inflammatory cytokines during the progression of inflammation. Numerous studies have shown that the Toll-like receptor-4 (TLR4) pathway is a major downstream signalling pathway for LPS. Notably, TLR4 is not only associated with microbial pathogenesis but also with multiple diseases such as IBD, autoimmune and allergic diseases [23-25]. Moreover, TLR4 is involved in the inflammation response, particularly in the secretion of inflammatory cytokines because it links several key inflammation-associated pathways such as NF- $\mathrm{KB}$, MAPK and IRF3[25-27]. As MIMP showed a significant inhibitory effect on LPS-induced inflammation, we hypothesized that MIMP may regulate inflammatory cytokines through affecting the TLR4 pathway. We first validated whether the TLR4 antagonist had the same regulatory effect as MIMP during LPS induction. As shown, see online suppl. material, in Fig. S8, the TLR4 antagonist, similar to MIMP, suppressed apical or basolateral secretion of IFN- $\gamma$, IL-17 and IL-23 induced by LPS and promoted the secretion of IL-4 and IL-10. Similarly, we observed that the TLR4 antagonist also inhibited the expression of IFN- $\gamma$, IL-17 and IL-23 induced by LPS, as well as up-regulated IL-4 and IL-10, in both apical and basolateral cells (see online suppl. material, Fig. S9).

Because MIMP and the TLR4 antagonist produce similar effects on inflammatory cytokines, we assumed that MIMP may have an impact on TLR4-associated downstream pathways during LPS induction. As expected, we observed that MIMP suppressed NF- $\kappa B$, 


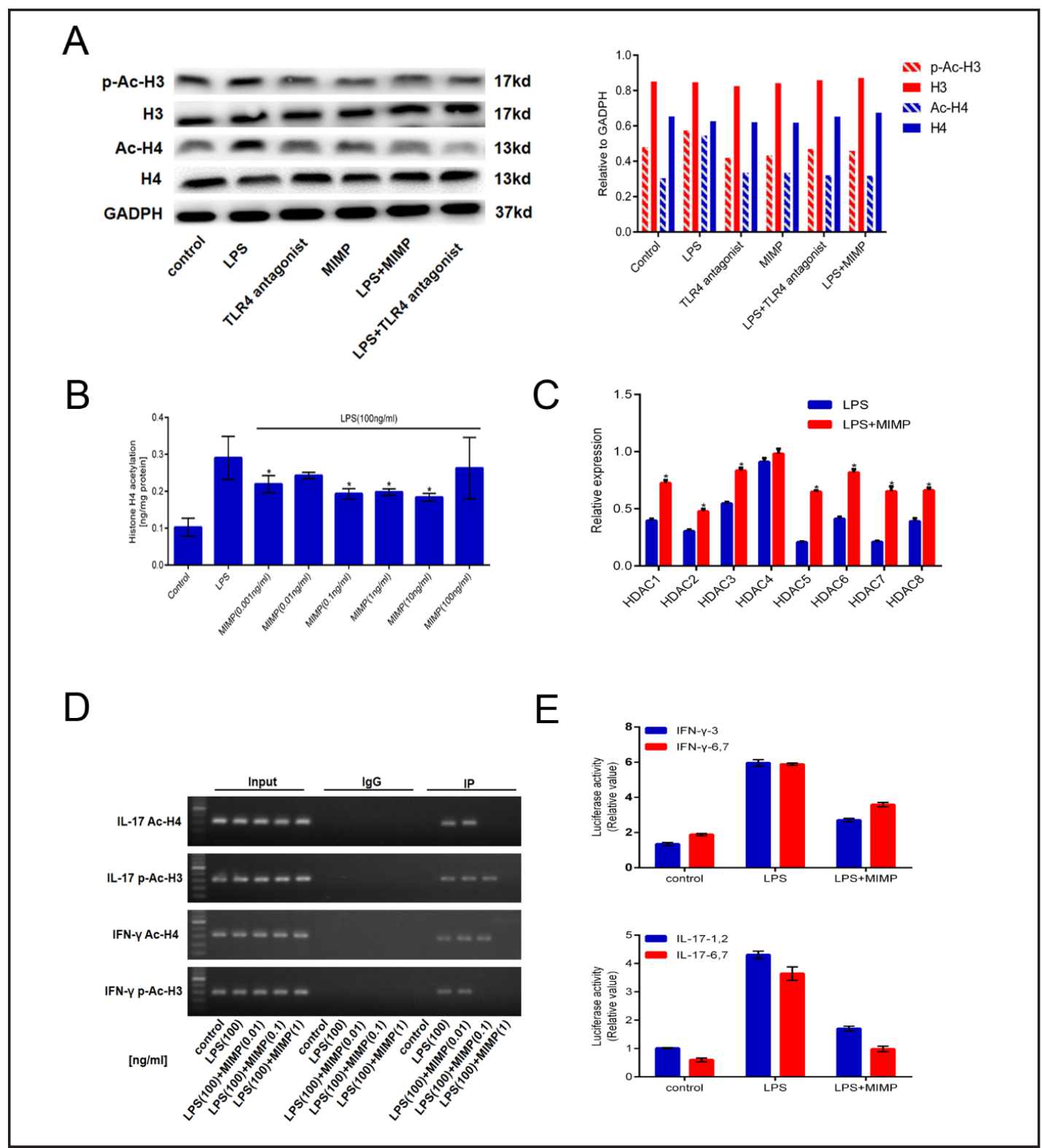

Fig. 6. MIMP regulates secretory inflammatory cytokines through Histone acetylation(A) LPS remarkably induced the acetylation of both $\mathrm{H} 3$ and $\mathrm{H} 4$ in CaCO-2 cells compared with that in the control group, while MIMP or the TLR4 antagonist reduced the acetylation level of H4 and H3 induced by LPS. (B) MIMP significantly inhibited LPS-induced histone $\mathrm{H} 4$ acetylation at the concentrations of $0.001 \mathrm{ng} / \mathrm{ml}, 0.1 \mathrm{ng} / \mathrm{ml}, 1 \mathrm{ng} /$ $\mathrm{ml}$ and $10 \mathrm{ng} / \mathrm{ml}$. (C) MIMP up-regulates the relative mRNA level of histone deacetylase (HDAC) 1-3 and HDAC5-8 in LPS-induced CaCO-2 cells. (D) LPS induces the acetylation of H3 and H4 in the promoter region of IFN- $\gamma$ and IL-17, while this effect is suppressed by MIMP intervention in a dose-dependent manner, with an optimal concentration of $1 \mathrm{ng} / \mathrm{ml}$. (E) MIMP significantly suppresses the promoter activity of IL-17 (upper) and IFN- $\gamma$ (bottom) induced by LPS. The data are expressed as the means \pm SD $\left({ }^{*} \mathrm{P}<0.05\right)$.

MAPK and JNK pathways activated by LPS in CaCO-2 cells (Fig. 5A and B). To further investigate whether the impact of MIMP on these pathways occurs in the early phase of LPS induction, we transfected CaCO-2 cells with NF- $\kappa \mathrm{B}$ luciferase reporter plasmids and subjected them to LPS or MIMP treatment with different incubation times. Compared with the TLR4 antagonist, MIMP had a limited effect on NF- $\kappa B$ activity at $6 \mathrm{~h}$ after MIMP treatment, although it began to exert a suppressive effect on NF- $\kappa B$ activity at $9 \mathrm{~h}$ and $12 \mathrm{~h}$ after treatment $(\mathrm{p}<0.05$, Fig. 


\section{Cellular Physiology Cell Physiol Biochem 2018;45:474-490 \begin{tabular}{ll|l} 
DOI: 10.1159/000487027 & O 2018 The Author(s). Published by S. Karger AG, Basel \\
www.karger.com/cpb
\end{tabular} \\ Yin et al.: MIMP Regulates Gut Barrier, Mcrobiota and Inflammatory Cytokines}

5C). Nevertheless, these findings suggested that MIMP inhibits the inflammation process by regulating the TLR4 pathway and its associated pathways.

Recent studies have shown gut microbiota and their metabolites could affect the host epigenome [28], suggesting that MIMP may also regulate the expression of inflammatory cytokines in an epigenetic manner. Because histone acetylation is closely associated with gene activation, we measured the global acetylation level of histone $\mathrm{H} 4$ or $\mathrm{H} 3$ in CaCO-2 cells treated with PBS (control), LPS, the TLR4 antagonist or MIMP. As shown in Fig. 6A, LPS remarkably induced the acetylation of both $\mathrm{H} 3$ and $\mathrm{H} 4$ in CaCO-2 cells compared with that in the control group. Interestingly, we observed that MIMP or TLR4 antagonist reduced the acetylation level of $\mathrm{H} 4$ and $\mathrm{H} 3$ induced by LPS. To further confirm this result, the acetylation assay was performed to quantify the acetylation level of $\mathrm{H} 4$ in CaCO-2-treated cells with a different dosage of MIMP. As shown in Fig. 6B, MIMP significantly inhibited LPS-induced histone $\mathrm{H} 4$ acetylation at the concentrations of $0.001 \mathrm{ng} / \mathrm{ml}, 0.1 \mathrm{ng} / \mathrm{ml}, 1 \mathrm{ng} / \mathrm{ml}$ and 10 $\mathrm{ng} / \mathrm{ml}(\mathrm{p}<0.05)$. Considering that histone deacetylases (HDACs) are important regulators of histone acetylation, we measured the expression level of HDAC1-8 in the control, LPStreated and MIMP-intervention group (Fig. 6C). We found several subtypes of HDAC, such as HDAC1-3 and HDAC5-8, were up-regulated by MIMP during LPS treatment, suggesting that the down-regulation of HDACs by MIMP treatment may contribute to the inhibition of LPSinduced histone acetylation.

We further investigated whether the global acetylation histone caused by MIMP intervention could affect the promoter activity of inflammatory cytokines. As shown in Fig. 4 , the expression level of IFN- $\gamma$ and IL-17 was significantly affected by MIMP during LPS treatment, compared with other inflammatory cytokines. We next evaluated the promoter activity of these two inflammatory cytokines in CaCO-2 cells after treatment with LPS or MIMP. We first evaluated the histone modification in the promoter region of IFN- $\gamma$ and IL-17 in CaCO-2 cells with different treatment. As shown in Fig. 6D, LPS could induce the acetylation of $\mathrm{H} 3$ and $\mathrm{H} 4$ in the promoter region of IFN- $\gamma$ and IL-17, while this effect was suppressed by MIMP intervention in a dose-dependent manner, suggesting that MIMP could affect LPS activation of IFN- $\gamma$ and IL-17 through histone modification. Similarly, we also observed that such a suppressive effect of MIMP on LPS was time-dependent (see online suppl. material, Fig. S10). To confirm the above results, we also constructed reporter plasmids containing different promoter regions of IL-17 and IFN- $\gamma$ (see online suppl. material, Fig. S11). Similarly, we observed that MIMP significantly suppressed the promoter activity of IL-17 and IFN- $\gamma$ induced by LPS ( $p<0.05$, Fig. $6 \mathrm{E}$ ). Collectively, our results, for the first time, demonstrate that MIMP can affect histone modification by LPS and, therefore, inhibit the transcription of proinflammatory cytokines IL-17 and IFN- $\gamma$.

\section{Discussion}

The beneficial role of LP in inducing IBD remission has been highly appreciated by accumulating studies $[29,30]$. Ours and other groups have found that SLP plays a crucial part in controlling inflammation, maintaining healthy gut microbiota and protecting the intestinal barrier [12,31]. Furthermore, for the first time, we isolated MIMP as the smallest domain of surface layer protein from Lactobacillus Plantarum and found its beneficial role in the protection of intestinal epithelial cells from enteropathogenic Escherichia Coli-induced injury [12]. In this study, we first established a DSS-induced colitis model, and we observed that MIMP intervention effectively controlled inflammation caused by DSS. Second, we found that MIMP alleviates DSS-treated mice from intestinal inflammation through protecting the gut barrier, modulating the microbiome and regulating inflammatory cytokines. Third, we showed that MIMP regulates the expression of inflammatory cytokines in PBMCs and CaCO2 cells in a time- and dose-dependent manner during LPS-induced inflammation. Finally, we reveal that the mechanisms by which MIMP modulates inflammatory cytokines expression occur through the TLR4 pathway and histone acetylation (Fig. 7). 


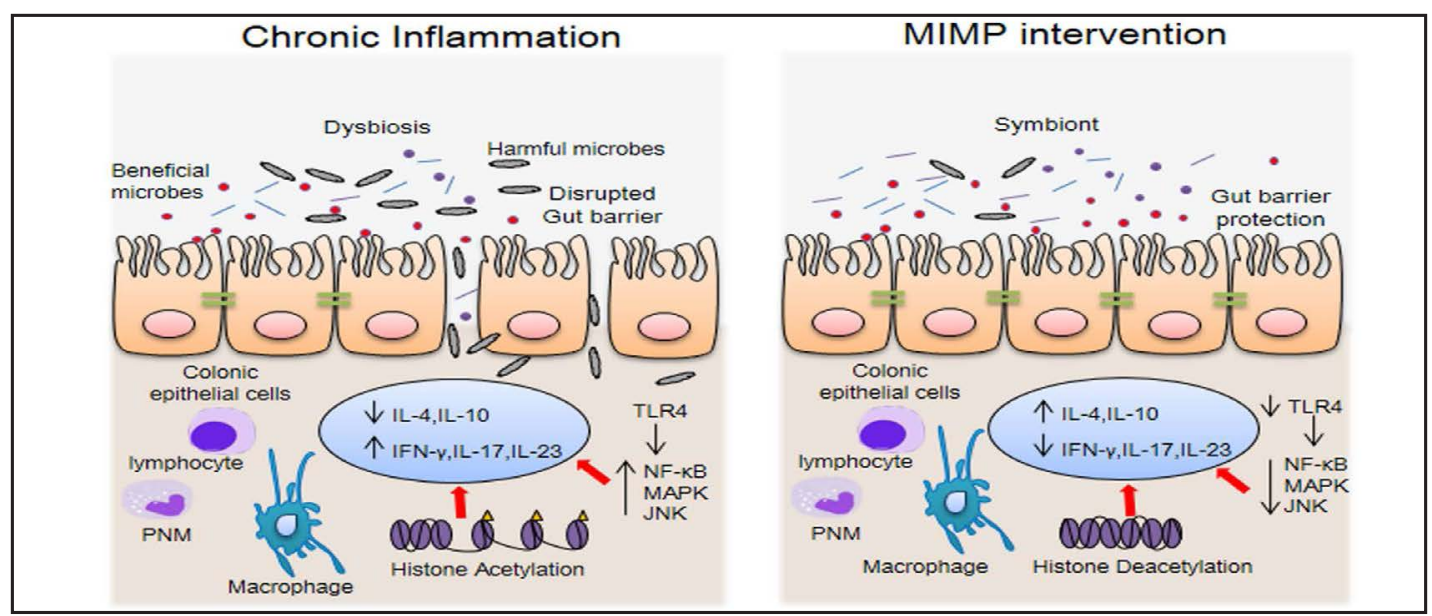

Fig. 7. Schematic summary for the intervening role of MIMP in chronic intestinal inflammationIn chronic intestinal inflammation (left), harmful microbes translocate into intestinal epithelium through the disrupted barrier. In addition, the secretion of pro-inflammatory cytokines (IFN- $\gamma$, IL-17 and IL-23) is increased, and the secretion of anti-inflammatory cytokines (IL-4 and IL-10) is decreased, functions associated with activated TLR-4-related signalling pathways and histone acetylation. After MIMP intervention, the intestinal epithelial barrier is protected, and gut microbiota dysbiosis is improved, characterized as increased beneficial microbes and decreased harmful microbes. Moreover, the TLR-4-related signalling pathways and histone acetylation are both inhibited, resulting in the decreased secretion of pro-inflammatory cytokines and increased secretion of anti-inflammatory cytokines. These changes collectively alleviate the progression of chronic intestinal inflammation.

In the DSS-induced colitis model, we found that MIMP invention effectively mitigates body weight loss, increases the colon length and reduces disease activity. The above antiinflammation effect was also confirmed by histological scoring and the expression level of pro- or anti-inflammatory cytokines in inflamed and control colon tissues, indicating that MIMP treatment is beneficial for controlling IBD progression. Because our previous work suggested MIMP can protect the intestine barrier from EPEC-induced injury, we next performed an in vivo permeability assay to validate whether MIMP also contributes to intestinal barrier protection in the DSS-induced colitis model. As expected, we observed that MIMP treatment dramatically decreased the FITC concentration in the peripheral blood of colitis mice, suggesting its potential role in the reduction of intestinal permeability. Consistent with the above results, we also found that the expression of tight junction molecules, such as JAM-1, Occludin and ZO-1, was significantly up-regulated in inflamed colon tissues. Because it is well recognized that IBD development is commonly associated with a leaky intestine barrier, leading to inflammation reaction and mucosal damage [32], we conclude that MIMP might serve as an important inhibitor of IBD by maintaining epithelial intercellular junctions during inflammation progression.

Numerous studies have proposed that probiotics improve the clinical outcome of IBD patients through influencing host microbiota [33]. Herein, we performed pyrosequencing analysis to investigate whether MIMP can improve the gut dysbiosis in the colitis model. As expected, we observed that DSS treatment caused significantly the decreased diversity of gut microbiota compared with the control group, a finding that is consistent with that in previous studies on IBD patients and the DSS-induced colitis model [34, 35]. In particular, MIMP intervention remarkably increased bacterial diversity and modified the community structure in the colitis mice, as shown by our cluster analysis and PCoA analysis. Specifically, we noticed that MIMP treatment dramatically decreased the abundance of Firmicutes, which were previously shown to be associated with IBD development [36-38]. Likewise, we observed a decreased trend in the abundance of pro-inflammatory microbiota in the MIMP-treated group, such as Lachnospiraceae and Allobaculum [38, 39]. In addition, Leuconostocaceae 


\section{Cellular Physiology Cell Physiol Biochem 2018;45:474-490 \\ \begin{tabular}{ll|l} 
DOI: 10.1159/000487027 & O 2018 The Author(s). Published by S. Karger AG, Basel \\
wwww.karger.com/cpb
\end{tabular} \\ Yin et al.: MIMP Regulates Gut Barrier, Mcrobiota and Inflammatory Cytokines}

and Leuconostoc, previously identified probiotics that exert anti-inflammatory effects by inducing IL-10 secretion [40], were enriched in the MIMP-treated group. Therefore, these findings provide novel evidence that MIMP plays an effective modulator of gut microbiota dysbiosis caused by intestinal inflammation.

Based on the above in vivo findings, we further investigated the influence of MIMP on inflammatory cytokines in PBMCs and the CaCO-2 co-culture model. We found that MIMP extremely decreased the levels pro-inflammation cytokines (IFN- $\gamma$, IL-17 and IL-23) but increased those of anti-inflammation cytokines (IL-4 and IL-10) in PBMCs and CaCO-2 cells. Moreover, such an effect was time- and dose-dependent, and MIMP exerted its maximal effect at the concentration of $1 \mathrm{ng} / \mathrm{ml}$ and after $12 \mathrm{~h}$ of treatment. The TLR4 antagonist is currently considered as an important inhibitor of LPS-induced inflammation [41]. Interestingly, MIMP has the similar anti-inflammation effect as the TLR4 antagonist, implying that MIMP may affect inflammatory cytokine expression through TLR4 pathways. Indeed, our results confirmed our assumption that MIMP significantly suppressed the TLR4 downstream MAPK/NF- $\kappa B$ signalling pathway activated by LPS treatment. Accumulating evidences have demonstrated that epigenetic modification plays a crucial role in the pathogenesis of IBD, particularly in histone acetylation [42]. Furthermore, histone acetylation was reported to be associated with the transcriptional activity of inflammatory cytokines [42, 43]. Herein, we found that MIMP intervention can suppress the LPS-induced globe acetylation level of histone $\mathrm{H} 3 / \mathrm{H} 4$ and increased the expression of histone deacetylases (HDACs). Notably, our ChIP assay showed that the increased acetylation level of histone $\mathrm{H} 3 / \mathrm{H} 4$ in the promoter region of IL-17 and IFN- $\gamma$ by LPS treatment was suppressed by MIMP treatment. A recent study reported that HDAC2 repressed the transcription of IL- 6 via histone deacetylation [44], suggesting that MIMP might repress IL-17 and IFN- $\gamma$ through recruiting HDACs to the promoter of IL-17 and IFN- $\gamma$, leading to the inhibition of their expression and relieved inflammation progression.

\section{Conclusion}

Our study, for the first time, demonstrated that MIMP serves as an inhibitor of inflammation by protecting the intestinal barrier, regulating inflammatory cytokines and improving gut microbiota dysbiosis. In vitro, MIMP was found to regulate the expression of inflammatory cytokines through the TLR4 pathway and histone modification. These findings firmly support and advocate the clinical translation of MIMP in the treatment of IBD.

\section{Acknowledgements}

This work was financially sponsored by grants from the National Natural Science Foundation Key projects of China (No.81230057; No.81472262; No.81372615), the National High Technology Research and Development Program (863 Program; No. 2014AA020803), Emerging cutting-edge technology joint research projects of Shanghai (No.SHDC12012106), Tongji University outstanding youth program (No. 1501219074), Tongji University key construction program (No. 162385) and Research Program of Wuxi hospital management center (No. YGZXM1531).

Study concept and design: M.Y., X.Y., W.W., H.Q. and Y.M.; Analysis and interpretation of data and statistical analysis: M.Y., X.Y., W.W., R.G., Q.W., Q.Z. and Y.M; In vitro experiments: M.Y., X.Y., M.L., C.P. and H.L.; Animal experiments: M.Y., Y.Y., T.S. and H.L.; Drafting of the manuscript: M.Y., X.Y., W.W., H.Q. and Y.M.

\section{Disclosure Statement}

The authors declare no competing financial interests. 


\section{Cellular Physiology Cell Physiol Biochem 2018;45:474-490

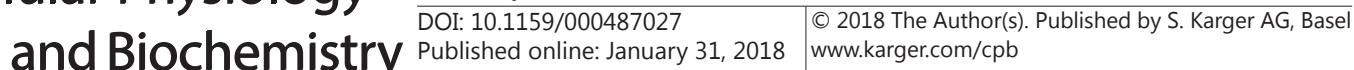 \\ Yin et al.: MIMP Regulates Gut Barrier, Mcrobiota and Inflammatory Cytokines}

\section{References}

1 Yang Y, Owyang C, Wu GD: East Meets West: The Increasing Incidence of Inflammatory Bowel Disease in Asia as a Paradigm for Environmental Effects on the Pathogenesis of Immune-Mediated Disease. Gastroenterology 2016;151:e1-e5.

2 Loddo I, Romano C: Inflammatory Bowel Disease: Genetics, Epigenetics, and Pathogenesis. Front Immunol 2015;6:551.

3 Chande N, Marshall JK, Seow CH, Sandborn WJ, Parker CE, Nelson S, Feagan BG: New Applications for Traditional Drugs in Inflammatory Bowel Disease: What Do Cochrane Reviews Tell Us? Inflamm Bowel Dis 2015;21:2948-2957.

-4 Chan HC, Ng SC: Emerging biologics in inflammatory bowel disease. J Gastroenterol 2017;52:141-150.

5 Grimm V, Riedel CU: Manipulation of the Microbiota Using Probiotics. Adv Exp Med Biol 2016;902:109-117.

6 Qin H, Zhang Z, Hang X, Jiang Y: L. plantarum prevents enteroinvasive Escherichia coli-induced tight junction proteins changes in intestinal epithelial cells. BMC Microbiol 2009;9:63.

7 Liu Z, Zhang P, Ma Y, Chen H, Zhou Y, Zhang M, Chu Z, Qin H: Lactobacillus plantarum prevents the development of colitis in IL-10-deficient mouse by reducing the intestinal permeability. Mol Biol Rep 2011;38:1353-1361.

8 Liu ZH, Shen TY, Zhang P, Ma YL, Moyer MP, Qin HL: Protective effects of Lactobacillus plantarum against epithelial barrier dysfunction of human colon cell line NCM460. World J Gastroenterol 2010;16:5759-5765.

-9 Liu Z, Ma Y, Shen T, Chen H, Zhou Y, Zhang P, Zhang M, Chu Z, Qin H: Identification of DC-SIGN as the receptor during the interaction of Lactobacillus plantarum CGMCC 1258 and dendritic cells. World J Microbiol Biotechnol 2010;27:603-611.

10 Liu ZH, Huang MJ, Zhang XW, Wang L, Huang NQ Peng H, Lan P, Peng JS, Yang Z, Xia Y, Liu WJ, Yang J, Qin HL, Wang JP: The effects of perioperative probiotic treatment on serum zonulin concentration and subsequent postoperative infectious complications after colorectal cancer surgery: a double-center and double-blind randomized clinical trial. Am J Clin Nutr 2013;97:117-126.

11 Liu Z, Shen T, Zhang P, Ma Y, Qin H: Lactobacillus plantarum surface layer adhesive protein protects intestinal epithelial cells against tight junction injury induced by enteropathogenic Escherichia coli. Mol Biol Rep 2011;38:3471-3480.

12 Liu Z, Shen T, Moyer MP, Qin H: Identification of the Lactobacillus SLP domain that binds gastric mucin. Front Biosci (Landmark Ed) 2011;16:2128-2143.

13 Liu Z, Ma Y, Yang J, Zhang P, Moyer MP, Qin H: Expression of the Lactobacillus plantarum surface layer MIMP protein protected NCM460 epithelial cells from enteroinvasive Escherichia coli infection. Cell Physiol Biochem 2011;27:99-108.

14 Liu Z, Shen T, Chen H, Zhou Y, Zhang P, Ma Y, Moyer MP, Zhang M, Chu Z, Qin H: Functional characterization of MIMP for its adhesion to the intestinal epithelium. Front Biosci (Landmark Ed) 2011;16:2106-2127.

15 Liu Z, Ma Y, Moyer MP, Zhang P, Shi C, Qin H: Involvement of the mannose receptor and p38 mitogenactivated protein kinase signaling pathway of the microdomain of the integral membrane protein after enteropathogenic Escherichia coli infection. Infect Immun 2012;80:1343-1350.

16 Liu Z, Kang L, Li C, Tong C, Huang M, Zhang X, Huang N, Moyer MP, Qin H, Wang J: Knockout of MIMP protein in lactobacillus plantarum lost its regulation of intestinal permeability on NCM460 epithelial cells through the zonulin pathway. BMC Gastroenterol 2014;14:171.

17 Jochems C, Fantini M, Fernando RI, Kwilas AR, Donahue RN, Lepone LM, Grenga I, Kim YS, Brechbiel MW, Gulley JL, Madan RA, Heery CR, Hodge JW, Newton R, Schlom J, Tsang KY: The ID01 selective inhibitor epacadostat enhances dendritic cell immunogenicity and lytic ability of tumor antigen-specific T cells. Oncotarget 2016;7:37762-37772.

18 Liu W, Guo W, Hang N, Yang Y, Wu X, Shen Y, Cao J, Sun Y, Xu Q: MALT1 inhibitors prevent the development of DSS-induced experimental colitis in mice via inhibiting NF-kappaB and NLRP3 inflammasome activation. Oncotarget 2016;7:30536-30549.

19 Mariman R, Kremer B, van Erk M, Lagerweij T, Koning F, Nagelkerken L: Gene expression profiling identifies mechanisms of protection to recurrent trinitrobenzene sulfonic acid colitis mediated by probiotics. Inflamm Bowel Dis 2012;18:1424-1433.

20 Chassaing B, Aitken JD, Malleshappa M, Vijay-Kumar M: Dextran sulfate sodium (DSS)-induced colitis in mice. Curr Protoc Immunol 2014;104:15-25.

-21 Carding S, Verbeke K, Vipond DT, Corfe BM, Owen LJ: Dysbiosis of the gut microbiota in disease. Microb Ecol Health Dis 2015;26:26191.

22 Stadnyk AW: Intestinal epithelial cells as a source of inflammatory cytokines and chemokines. Can J 


\section{Cellular Physiology Cell Physiol Biochem 2018;45:474-490 \begin{tabular}{ll|l} 
and Biochemistry 10.1159/000487027 & $\begin{array}{l}\text { Published on } 2018 \text { The Author(s). Published by S. Karger AG, Basel } \\
\text { www.karger.com/cpb }\end{array}$ \\
\hline
\end{tabular}}

Gastroenterol 2002;16:241-246.

23 Liaunardy-Jopeace A, Gay NJ: Molecular and cellular regulation of toll-like receptor-4 activity induced by lipopolysaccharide ligands. Front Immunol 2014;5:473.

-24 Kamba A, Lee IA, Mizoguchi E: Potential association between TLR4 and chitinase 3-like 1 (CHI3L1/YKL-40) signaling on colonic epithelial cells in inflammatory bowel disease and colitis-associated cancer. Curr Mol Med 2013;13:1110-1121.

25 Gyires K, Toth EV, Zadori SZ: Gut inflammation: current update on pathophysiology, molecular mechanism and pharmacological treatment modalities. Curr Pharm Des 2014;20:1063-1081.

26 Rocha DM, Caldas AP, Oliveira LL, Bressan J, Hermsdorff HH: Saturated fatty acids trigger TLR4-mediated inflammatory response. Atherosclerosis 2016;244:211-215.

-27 Liu Y, Yin H, Zhao M, Lu Q: TLR2 and TLR4 in autoimmune diseases: a comprehensive review. Clin Rev Allergy Immunol 2014;47:136-147.

28 Mischke M, Plosch T: The Gut Microbiota and their Metabolites: Potential Implications for the Host Epigenome. Adv Exp Med Biol 2016;902:33-44.

29 Curro D, Ianiro G, Pecere S, Bibbo S, Cammarota G: Probiotics, fibre and herbal medicinal products for functional and inflammatory bowel disorders. Br J Pharmacol 2017;174:1426-1449.

-30 Fedorak RN, Feagan BG, Hotte N, Leddin D, Dieleman LA, Petrunia DM, Enns R, Bitton A, Chiba N, Pare P, Rostom A, Marshall J, Depew W, Bernstein CN, Panaccione R, Aumais G, Steinhart AH, Cockeram A, Bailey RJ, Gionchetti P, Wong C, Madsen K: The probiotic VSL\#3 has anti-inflammatory effects and could reduce endoscopic recurrence after surgery for Crohn's disease. Clin Gastroenterol Hepatol 2015;13:928-935.

-31 Lightfoot YL, Selle K, Yang T, Goh YJ, Sahay B, Zadeh M, Owen JL, Colliou N, Li E, Johannssen T, Lepenies B, Klaenhammer TR, Mohamadzadeh M: SIGNR3-dependent immune regulation by Lactobacillus acidophilus surface layer protein A in colitis. EMBO J 2015;34:881-895.

-32 Luissint AC, Parkos CA, Nusrat A: Inflammation and the Intestinal Barrier: Leukocyte-Epithelial Cell Interactions, Cell Junction Remodeling, and Mucosal Repair. Gastroenterology 2016;151:616-632.

33 Sanchez B, Delgado S, Blanco-Miguez A, Lourenco A, Gueimonde M, Margolles A: Probiotics, gut microbiota, and their influence on host health and disease. Mol Nutr Food Res 2017;61.

-34 Imhann F, Vich Vila A, Bonder MJ, Fu J, Gevers D, Visschedijk MC, Spekhorst LM, Alberts R, Franke L, van Dullemen HM, Ter Steege RW, Huttenhower C, Dijkstra G, Xavier RJ, Festen EA, Wijmenga C, Zhernakova A, Weersma RK: Interplay of host genetics and gut microbiota underlying the onset and clinical presentation of inflammatory bowel disease. Gut 2016;206:2016-312135.

35 Zhang Q, Wu Y, Wang J, Wu G, Long W, Xue Z, Wang L, Zhang X, Pang X, Zhao Y, Zhao L, Zhang C: Accelerated dysbiosis of gut microbiota during aggravation of DSS-induced colitis by a butyrate-producing bacterium. Sci Rep 2016;6:27572.

-36 Vital M, Howe AC, Tiedje JM: Revealing the bacterial butyrate synthesis pathways by analyzing (meta) genomic data. MBio 2014;5:e00889.

-37 Labbe A, Ganopolsky JG, Martoni CJ, Prakash S, Jones ML: Bacterial bile metabolising gene abundance in Crohn's, ulcerative colitis and type 2 diabetes metagenomes. PLoS One 2014; 9:e115175.

38 Zhang Z, Wu X, Cao S, Wang L, Wang D, Yang H, Feng Y, Wang S, Li L: Caffeic acid ameliorates colitis in association with increased Akkermansia population in the gut microbiota of mice. Oncotarget 2016;7:31790-31799.

-39 Lee SM, Han HW, Yim SY: Beneficial effects of soy milk and fiber on high cholesterol diet-induced alteration of gut microbiota and inflammatory gene expression in rats. Food Funct 2015;6:492-500.

-40 Latvala S, Miettinen M, Kekkonen RA, Korpela R, Julkunen I: Lactobacillus rhamnosus GG and Streptococcus thermophilus induce suppressor of cytokine signalling 3 (SOCS3) gene expression directly and indirectly via interleukin-10 in human primary macrophages. Clin Exp Immunol 2011;165:94-103.

41 Malgorzata-Miller G, Heinbockel L, Brandenburg K, van der Meer JW, Netea MG, Joosten LA: Bartonella quintana lipopolysaccharide (LPS): structure and characteristics of a potent TLR4 antagonist for in-vitro and in-vivo applications. Sci Rep 2016;6:34221.

42 Ventham NT, Kennedy NA, Nimmo ER, Satsangi J: Beyond gene discovery in inflammatory bowel disease: the emerging role of epigenetics. Gastroenterology 2013;145:293-308.

43 Meng J, Liu X, Zhang P, Li D, Xu S, Zhou Q, Guo M, Huai W, Chen X, Wang Q Li N, Cao X: Rb selectively inhibits innate IFN-beta production by enhancing deacetylation of IFN-beta promoter through HDAC1 and HDAC8. J Autoimmun 2016;73:42-53.

44 Zhang Q, Zhao K, Shen Q Han Y, Gu Y, Li X, Zhao D, Liu Y, Wang C, Zhang X, Su X, Liu J, Ge W, Levine RL, Li $\mathrm{N}$, Cao X: Tet2 is required to resolve inflammation by recruiting Hdac2 to specifically repress IL-6. Nature 2015;525:389-393. 